\title{
A ALOCAÇÃo ESTRATÉGICA DE RECURSOS INSTITUCIONAIS PARA A PóS- GRADUAÇÃO STRICTO SENSU E O DESEMPENHO DOS PROGRAMAS NA AVALIAÇÃO CAPES: UM ESTUDO EM UNIVERSIDADES FEDERAIS
}

Recebido: 14/06/2019

Elida Furtado da Silva Andrade ${ }^{1}$ Josiane de Oliveira Lobato ${ }^{2}$ Emerson Antonio Maccari ${ }^{3}$ Aprovado: $23 / 09 / 2019$ Marcos Rogério 4

\section{Resumo}

As universidades públicas com importante atuação na pós-graduação stricto sensu têm, ao longo dos anos, procurado definir estratégias para alavancar o desempenho dos seus programas por meio de ações internas que os fortaleçam diante dos critérios exigidos na avaliação periódica realizada pela CAPES. Este estudo pretende analisar como as próreitorias de pós-graduação de universidades federais alocaram recursos institucionais em busca do desenvolvimento de seus programas de pós-graduação stricto sensu, no período de 2008 a 2017. Nesse sentido, foi realizado um estudo de caso múltiplo, utilizando como unidades de análise a Universidade Federal de Mato Grosso/UFMT, a Universidade Federal do Amazonas/UFAM e a Universidade Federal de Mato Grosso do Sul/UFMS. Os dados primários e secundários, respectivamente, foram obtidos por meio de entrevistas com as pró-reitoras de pós-graduação stricto sensu das universidades investigadas e em sites e documentos oficiais disponibilizados pelas instituições, observando os preceitos da teoria Resource Based-view/RBV. Com os resultados da pesquisa, concluiu-se ser imprescindível para a expansão e/ou consolidação da pós-graduação stricto sensu, ofertadas pelas universidades federais, o seu reconhecimento pela administração superior como estratégica para o desenvolvimento institucional, fazendo-a constar no PDI e, respectivamente, nos planejamentos estratégicos de curto/médio prazos propostos pela gestão. Esse registro asseguraria a alocação de seus recursos institucionais utilizando sua experiência acumulada e de acordo com as políticas institucionais definidas em seu planejamento estratégico.

Palavras-chave: Avaliação. Pós-graduação. RBV. Estratégia.

\section{Como Citar:}

Andrade, E., Lobato, J., Maccari, E., \& Mazieri, M. (2020). A Alocação Estratégica de Recursos Institucionais para a Pós-Graduação Stricto Sensu e o Desempenho dos Programas na Avaliação Capes: Um Estudo em Universidades Federais. Future Studies Research Journal: Trends and Strategies [FSRJ], 12(1), 175-202. doi:https://doi.org/10.24023/FutureJournal/2175-5825/2020.v12i1.444

\footnotetext{
1Universidade Nove de Julho - UNINOVE, São Paulo, (Brasil). E-mail: elidandrade@gmail.com Orcid id: https://orcid.org/0000-0001-7064-7633

2Universidade Nove de Julho - UNINOVE, São Paulo, (Brasil). E-mail: josiane deoliveira@hotmail.com Orcid id: https://orcid.org/0000-0002-4774-183X

3Universidade Nove de Julho - UNINOVE, São Paulo, (Brasil). E-mail: emersonmaccari@gmail.com Orcid id: http://orcid.org/0000-0001-7085-224X

${ }^{4}$ Universidade Nove de Julho - UNINOVE, São Paulo, (Brasil). E-mail: marcosmazzzieri@gmail.com Orcid id: https://orcid.org/0000-0003-1338-3912
} 


\begin{abstract}
Public universities with impacting actions in postgraduate studies have, throughout the years, tried to define strategies to leverage their programs performances through internal actions that may strengthen them before the criteria demanded in the periodical evaluation performed by CAPES. This study intends to analyze how the postgraduate rectory of federal universities allocates organizational resources when seeking the development of its postgraduate programs, from 2008 to 2017. In this respect, a multiple case study was conducted, using the federal universities of the State of Mato Grosso/UFMT, State of Amazonas/UFAM and Mato Grosso do Sul/UFMS as analysis units. The primary and secondary data, respectively, were obtained by interviewing the postgraduate deans of the researched universities and also on websites and official documents available, observing the precepts of the Resource Based-view Theory. With the research results we concluded that is indispensable for the expansion and/or consolidation of the postgraduate programs offered by federal universities, their recognition by the higher administration as strategic programs for organizational development, by including them in the Internal Development Program and, respectively, on strategic planning of short/medium term proposed by the administration. This registry would assure the allocation of organizational resources according to the gained expertise and also according to institutional policies defined for the strategic planning.
\end{abstract}

Keywords: Evaluation. Postgraduate. RBV. Strategy. 


\section{INTRODUÇÃO}

No período de 2007 a 2017, o Sistema Nacional de Pós-Graduação/SNPG cresceu em torno de $85,7 \%$, praticamente o mesmo percentual de crescimento que o sistema conseguiu atingir nos no período de 1975 a 1998, ou seja 23 anos, sendo que os maiores percentuais de expansão se concentraram nos cursos de doutorado e mestrado profissional. Esse resultado reflete o cumprimento das metas estabelecidas desde o I Plano Nacional de Pós-graduação/PNPG que visavam o crescimento, com qualidade, do Sistema Nacional de Pós-graduação/SNPG.

Esse crescimento constante na pós-graduação stricto sensu, observado desde o início do processo de avaliação implantado pela Coordenação de Aperfeiçoamento de Pessoal de Nível Superior/CAPES em 1976, corroboram a importância da pósgraduação stricto sensu para o país, na formação de profissionais altamente qualificados, aptos a responder às demandas do mercado e do mundo acadêmico, contribuindo, dessa forma, para o desenvolvimento tecnológico, científico e cultural da nação (Brasil, 2014).

Entre os anos de 2008 e 2015, vivenciou-se um crescimento considerável nas ações de indução buscando diminuir as dificuldades no setor da pós-graduação stricto sensu. Essas ações resultaram de um forte incremento no orçamento da CAPES e foram concretizadas por meio do aumento de concessões de bolsas e publicação de editais de fomento à pesquisa, alcançando e superando as metas previstas no PNPG vigente até 2020 (CAPES, 2017). Entretanto, em 2014, o país iniciou uma grave crise econômica, tendo como consequência contingenciamento orçamentário para todos os ministérios, inclusive o da Educação, chegando em 2017 a uma drástica redução no orçamento destinado à CAPES, impossibilitando, dessa forma, a continuidade de programas de indução à pesquisa e forçando a redução de financiamento de outros projetos, mesmo sendo de áreas consideradas estratégicas (CAPES, 2017).

A crise também atingiu as universidades federais que, entre 2007 e 2013, tiveram uma fase de reforço orçamentário, resultado de projetos de interiorização e expansão, como o plano de Reestruturação e Expansão das Universidades Federais/REUNI, tiveram seu orçamento contingenciados a partir de 2014, atingindo valores, em 2015 e 2016, próximos aos investidos no ano de 2007.

Mesmo diante de dificuldades orçamentárias, universidades públicas com atuação importante na pós-graduação stricto sensu têm, ao longo dos anos, procurado definir estratégias para alavancar o desempenho dos seus programas por intermédio de ações internas que os fortaleçam diante dos critérios exigidos pelas avaliações de áreas e pelo CTC/ES da CAPES (Martins, Maccari, Storopoli \& Andrade, 2013). 
Assim, considerando os esforços despendidos nessa direção, objetiva-se com este estudo analisar como as pró-reitorias de pós-graduação de universidades federais alocaram recursos institucionais em busca do desenvolvimento de seus programas de pós-graduação stricto sensu, no período de 2008 a 2017. Entende-se por "recursos institucionais" o conjunto de recursos que reúnem as parcerias firmadas em busca da internacionalização e cooperação com outros programas de pós-graduação, os recursos humanos (docentes, discentes e técnicos-administrativos), os recursos físicos (infraestrutura de salas de aula, laboratórios, bibliotecas, entre outros), os recursos tecnológicos (computadores, redes, antenas, sistemas, entre outros), os recursos organizacionais (rotinas, processos internos, gestão e uso de tecnologias, planejamento, entre outros), os recursos reputacionais (imagem, reputação, relacionamento com a comunidade interna e externa, entre outros) e os recursos financeiros (Barney, 1986; Dierickx ; Cool, 1989).

Para viabilizar essa pesquisa foi realizado um estudo de caso múltiplo, utilizando como unidades de análise a Universidade Federal de Mato Grosso/UFMT, a Universidade Federal do Amazonas/UFAM e a Universidade Federal de Mato Grosso do Sul/UFMS.

Para a análise dados primários e secundários, obtidos por meio de entrevistas com as pró-reitoras de pós-graduação stricto sensu das universidades investigadas, bem como em sites e documentos oficiais disponibilizados pelas universidades, observando os preceitos da teoria Resource Based-view/RBV. Essa teoria defende que o desempenho de uma empresa depende da forma como administra e utiliza seus recursos, admitindo que as empresas têm naturezas distintas e os recursos não são transferidos facilmente, pois sua distribuição na indústria não é uniforme (Barney, 1986).

Esse trabalho limitou-se a investigar o uso dos recursos institucionais para a execução de estratégias voltadas para o desenvolvimento da pós-graduação stricto sensu em três universidades federais brasileiras, no entanto, seus resultados podem contribuir com outras universidades, especialmente as federais, no processo de expansão e consolidação de sua pós-graduação stricto sensu na avaliação periódica conduzida pela CAPES.

A estrutura desse estudo está organizada em cinco seções, sendo a primeira essa introdução, seguido pelo referencial teórico, a metodologia, a apresentação e discussão dos resultados e, por fim, a conclusão do trabalho. 


\section{A PÓS-GRADUAÇÃO STRICTO SENSU NO BRASIL}

A pós-graduação stricto sensu brasileira é reconhecida nacional e internacionalmente como pilar na qualificação de pessoal altamente especializado, e considerada estratégica pelo governo para o desenvolvimento científico, educacional e tecnológico do país (Boratim, 2014). Para melhor compreensão do que isso significa, é importante nos reportarmos ao longo processo pelo qual passaram as instituições de ensino superior no Brasil até chegarmos ao ponto de ser possível analisar o sistema de avaliação da CAPES, considerado fundamental para o crescimento da pósgraduação stricto sensu no país.

Foi tardia a implementação da educação superior no Brasil, em relação a outras nações europeias e latino americanas. Somente em 1920, após intensos debates, o Brasil conheceu sua primeira universidade, a Universidade do Rio de Janeiro, nascida com a junção das já existentes Escolas de Medicina, Escola Politécnica e Faculdade de Direito; entretanto, essa junção de escolas não resultou em integração acadêmica dessas instituições, que continuaram suas atividades de forma independente, sem nenhuma conexão curricular ou de formação (Mendonça, 2000).

A reforma Francisco Campos, aprovada em 1931, possibilitou às universidades o estabelecimento de intercâmbios com instituições internacionais de ensino e pesquisa, atraindo professores estrangeiros, que vieram para o país em missões acadêmicas ou mesmo como asilados de guerra (pedidos feitos por profissionais europeus durante o período que precedeu a II Guerra mundial); esses profissionais ajudaram a implementar a pós-graduação stricto sensu no país, ancorada em padrões europeus, principalmente o francês, no qual cabia ao professor, autoridade maior na relação tutorial, determinar os caminhos a serem percorridos pelos estudantes para a conclusão do projeto de tese. Estas experiências tiveram pouca expressão para o ensino superior em nível nacional, pois ficavam circunscritas a um seleto grupo de indivíduos especialmente destinados à carreira docente, sendo o título de "doutor", até então, ainda desconhecido no mercado brasileiro (Balbachevsky, 2005).

Em 1961, embora já prevista na Constituição de 1946, foi publicada a Lei de Diretrizes e Bases da Educação/LDB, e por ela o sistema de ensino no Brasil passou a se organizar nos seguintes moldes: Educação Pré-primária, Ensino Primário, Ensino Médio, e o Ensino Superior (Brasil, 2018b). A LDB conferiu à pós-graduação grau específico de formação, o que foi regulamentado posteriormente com a publicação do parecer 977/1965, do Conselho Federal de Educação/CFE, relatado pelo professor Newton Sucupira. 
Pelo parecer Sucupira, a pós-graduação stricto sensu brasileira adotava o modelo norte-americano, estabelecendo os níveis de mestrado e doutorado, proficiência em língua estrangeira, exigência de cumprimento de créditos e defesa para obtenção do título, além de determinar tempo mínimo para conclusão do curso: um ano para o mestrado e dois para o doutorado (Cury, 2005). A LDB instituiu também a exigência de diploma de graduação para o ingresso na pós-graduação.

Em 1965, Newton Sucupira afirmou que a falta de tradição e experiência do Brasil na área estava levando a uma confusão na definição da natureza da pós-graduação pelas universidades, ao não especificar sua natureza, devido à falta de regulamentação sobre seu funcionamento, o que provocava visões distorcidas sobre a pós-graduação, fugindo da intenção inicial de distinguir os cursos como uma categoria que, unindo ensino e pesquisa, tinha por objetivo formar pesquisadores e docentes para a graduação (Almeida Júnior et al., 2005). No mesmo ano, o ministro da Educação e Cultura, Flávio Suplicy de Lacerda, enviou um aviso ministerial ao CFE solicitando uma conceituação e, até mesmo, uma regulamentação para os cursos de pós-graduação citados no art. 69 da LDB/1961.

Assim, o CFE escolheu o conselheiro Newton Sucupira como relator, cujo trabalho resultou no parecer 977/65, mais conhecido como Parecer Sucupira, aprovado em 03 de dezembro de 1965. Em seu trabalho, o relator destaca a importância da pósgraduação para o progresso da ciência em todas as áreas e a impossibilidade de levar este conhecimento, com profundidade, somente com estudos de graduação, (Almeida Júnior et al., 2005). Segundo Almeida Júnior et al. (2005), "a pós-graduação tornase, na universidade moderna, cúpula dos estudos, sistema especial de cursos exigido pelas condições da pesquisa científica e pelas necessidades do treinamento avançado".

O parecer 977/65 distinguiu os cursos de pós-graduação em lato sensu e stricto sensu: os primeiros afeitos à formação técnico-profissional específica, sem regularidade de oferta; e os segundo com objetivos de formação científica e cultural mais amplos e profundos, fazendo parte da estrutura da universidade, com oferta regular de cursos e concessão de grau acadêmico (Almeida Júnior et al., 2005).

No esforço para disciplinar a pós-graduação, o relator utilizou como modelo a pós-graduação norte-americana, cuja estrutura possuía dois ciclos e conferia dois graus, Master (mestre) e Doctor (doutor), e no qual existiam duas formas de doutorado e mestrado - o de pesquisa e o profissional. O Parecer Sucupira constituise na base dos princípios regimentares que norteiam, desde então, a pós-graduação, influenciando as normatizações posteriormente publicadas, além de amparar soluções para questões suscitadas na criação de programas (Almeida Júnior et al., 2005). 
Para Cury (2005), parece "não haver nenhum outro texto que articule doutrina e normatização sobre o assunto com tanto impacto sobre esse nível da educação superior no Brasil" quanto o parecer 977/65. Resultado da normatização, ainda em 1965, dos 38 cursos de pós-graduação stricto sensu existentes, 27 foram classificados no nível de "mestrado" e 11 no nível de doutorado (ACS/CAPES; Assessoria de Comunicação Social da CAPES, 2011).

A CAPES, criada em 1951 com o objetivo de estimular a formação de pessoal qualificado em áreas específicas, altamente necessário para o desenvolvimento nacional, teve sua estrutura alterada com a publicação do Decreto 74.299/1974, passando a ser um órgão central superior com autonomia financeira e administrativa, fazendo constar, entre suas finalidades, a de colaborar com a execução da Política Nacional de Pós-graduação (Brasil, 2018a).

Em 1974, foi publicado o I PNE, onde a pós-graduação stricto sensu assume posição estratégica para o país, tornando-se responsável pela formação de pesquisadores, professores para o magistério universitário e de profissionais altamente qualificados para o mercado de trabalho (França, 2012). Com vistas a atender às diretrizes preconizadas pelo I PNE, em 1976, a CAPES inicia o processo de avaliação da pós-graduação stricto sensu, com o intuito de aferir o nível de qualidade dos cursos ofertados para qualificação de pessoal necessário à expansão e consolidação de cursos ofertados no país (CAPES, 2017a).

Em uma visão mais ampla, o sistema de avaliação da CAPES busca identificar ações que levam os programas de pós-graduação stricto sensu a atingirem padrões de excelência internacional e, em uma visão mais interna, contribui para que os programas consigam tomar decisões relativas a autorizações e credenciamentos dos cursos, bem como na distribuição dos recursos para atingirem os resultados desejados (Belloni, 2000). O sistema de avaliação da CAPES se consolidou e vem se aperfeiçoando, revendo critérios e indicadores, e tem contribuído para o avanço das pesquisas científicas e tecnológicas no Brasil (Maccari, Rodrigues, Alessio, \& Quoniam, 2008).

Entre os anos 2008 e 2016, todas as regiões geográficas do país tiveram um crescimento relativo considerável na oferta de cursos de pós-graduação stricto sensu, figura 1, destacando-se neste cenário a região nordeste, que alcançou, por meio dos incentivos à formação de doutores, ofertar um número de programas de pósgraduação stricto sensu similar ao da região sul, ingressando, desse modo, no rol de regiões com número de programas de pós-graduação equivalentes, sem assimetrias relevantes (Geocapes, 2017). 


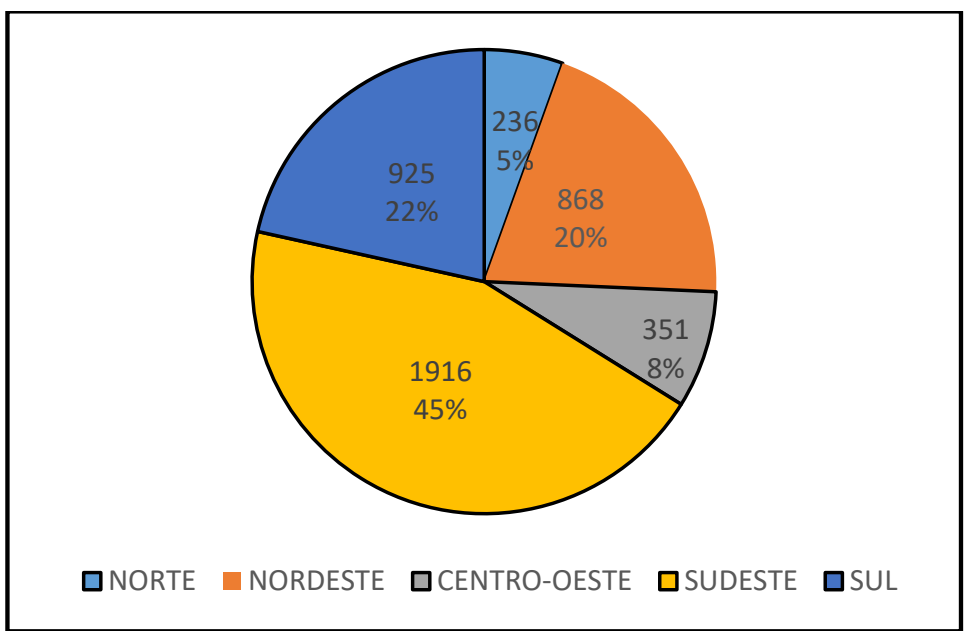

Figura 1 - Oferta de programas de pós-graduação stricto sensu por região 2017 Fonte: Geocapes

Em 2017, as universidades federais eram responsáveis pela oferta de cerca de $57 \%$ dos programas de pós-graduação stricto sensu existentes no país. Ribeiro (2007b) afirma que o processo de avaliação gera um ambiente altamente competitivo, devido a análise comparativa realizada entre as áreas e, no seu interior, entre os programas que a compõem.

Desde 2013, o orçamento das universidades vem sofrendo um contingenciamento aproximado de $40 \%$ dos recursos de capital (para obras e compra de equipamentos) e de até $60 \%$ dos recursos de custeio, exigindo de seus gestores uma administração mais eficiente e eficaz dos seus recursos e capacidades (BRASIL, 2017). Nesse processo, a teoria da Resource Based-View/RBV pode atuar como instrumento auxiliar na definição de estratégias na alocação dos recursos e capacidades internas das universidades federais, uma vez que, utilizada em diferentes contextos, possibilita uma compreensão mais ampla de seus objetivos (Pereira \& Forte, 2008).

\subsection{RESOURCE BASED-VIEW/RBV}

O termo Resource Based-view, doravante denominada de RBV, foi utilizado pela primeira vez por Wernerfelt (1984) em seu trabalho intitulado A Resource based view of the firm, cujo objetivo visava analisar as empresas pelo prisma dos seus recursos, bem como as opções estratégicas que estes podem oferecer. O autor suportou sua teoria nos escritos de Penrose, que em 1959, afirmou que o crescimento das empresas não residia no mercado, mas resultavam de seu conjunto de recursos e a forma com que estes eram operacionalizados.

A visão do uso dos recursos internos da firma para obter a vantagem competitiva, defendida na RBV, pode complementar o modelo ambiental defendido por Porter 
(1980), que observa a relação entre o ambiente externo da empresa, sua estratégia e seu desempenho (Mello \& Cunha, 2000; Ribeiro, Rossetto, \& Verdinelli, 2011). Entretanto, a RBV admite que as empresas têm naturezas distintas e os recursos não são transferidos facilmente, pois sua distribuição na indústria não é uniforme, contrapondo a teoria de Porter, onde as empresas são homogêneas em termos de recursos estratégicos (Bandeira-de-Mello \& Cunha, 2000).

O conceito de recursos foi ampliado por Barney (1991), atribuindo uma função específica, a de habilitar a empresa a elaborar e implementar estratégias que aperfeiçoem sua efetividade. Segundo o autor, recursos não utilizados no processo estratégico, não devem ser considerados recursos apenas ativos da empresa. Assim, o autor define que, na RBV, recursos são todos "os ativos, capacidades, processos organizacionais, conhecimento, informação e atributos controlados pela empresa" que a possibilite adotar estratégias que a torne mais efetiva (Barney, 1991).

Para dar origem a vantagem competitiva, os recursos devem possuir características específicas, segundo Barney (1991), descritas no modelo VRIS:

$\checkmark$ Valiosos (V) - devem proporcionar a criação de valor, estando aliados ao aumento das oportunidades e/ou redução das ameaças, com consequente redução de custos ou aumento de receitas;

$\checkmark \operatorname{Raros}(\mathrm{R})$ - ou escassos, quando comparados com a concorrência, expressa "raridade", pois estes recursos não devem pertencer a um número de empresas suficiente para gerar uma concorrência perfeita;

$\checkmark$ Imperfeitamente Imitáveis (I) - ainda que os recursos sejam valiosos e raros, para serem fonte de vantagem competitiva, a sua imitação pelos concorrentes seria tão custosa que inviabilizaria o processo. Segundo Barney (1986), o alto custo da imitação recai sobre uma ou pelo conjunto das seguintes explicações:

- Condições históricas únicas: a obtenção ou desenvolvimento deste recurso envolve condições de tempo e espaço que não podem ser recriados, pois são únicos, representando uma grande desvantagem para os concorrentes em termos de custo;

- Ambiguidade causal: ocorre quando a relação entre os recursos administrados pela empresa e a vantagem competitiva não são assimilados, ou seja, torna-se difícil a percepção da obtenção da vantagem competitiva por meio da gestão dos recursos da empresa. Entretanto, para que ocorra vantagem competitiva por meio da ambiguidade causal, nenhuma empresa deve compreender esta relação, caso contrário, a longo prazo, este conhecimento será difundido, extinguindo a ambiguidade causal; 
- Complexidade Social: alguns recursos e capacidades da empresa podem representar fenômenos sociais complexos, restringindo significativamente a possibilidade de imitação desses recursos ou capacidades pela concorrência. A reputação, a cultura e as relações interpessoais são exemplos de recursos socialmente complexos que, quando não perfeitamente imitáveis, agregadas às demais características (valiosos, raros e insubstituíveis), são fonte de vantagem competitiva.

$\checkmark$ Substituibilidade (S) - os recursos podem ser substitutos quando, mesmo sendo diferentes entre si, permitem a implantação de uma mesma estratégia ou atribuem a mesma capacidade às empresas. A imitação de um recurso pode ser imperfeita, no entanto, o concorrente pode substituí-lo por um recurso equivalente para idealizar e implementar as mesmas estratégias. Também recursos diferentes de um concorrente podem ser considerados substitutos, quando utilizados de forma estratégica.

Avançando nos estudos para avaliar os recursos na obtenção da vantagem competitiva, Peteraf (1993) apresenta as características essenciais que os recursos devem apresentar para atender este objetivo (Bandeira-de-Mello \& Cunha, 2000):

$\checkmark$ Heterogeneidade de Recursos: distribuição heterogênea de recursos. Somente as empresas detentoras dos recursos escassos e com eficiência superior alcançam a vantagem competitiva.

$\checkmark$ Mobilidade Imperfeita: refere-se a recursos de difícil transferência ou comercialização, pois são específicos da empresa, sem ou com pouco valor para uso fora dela.

$\checkmark$ Barreiras à Competição (ex ante): os recursos adquiridos ou acumulados pela empresa devem ser de difícil imitação ou substituição pela concorrência. Referem-se a imperfeições de mercado, informações privilegiadas, imagem da firma, ou seja, recursos que conferem condições diferenciadas para a empresa.

$\checkmark$ Barreiras à Competição (ex post): a posse de recursos de alto nível pela empresa, não deve provocar sua competição com a concorrência. Essas barreiras estão muito difíceis de imitar, pois são permeadas de conhecimento tácito adquirido e acumulado por meio da história organizacional, socialmente complexos e geram ambiguidade causal.

Assim, os recursos vitais para a vantagem competitiva fazem parte da experiência acumulada pela empresa no decorrer de sua existência, baseada em suas escolhas (Barney, 1986; Dierickx ; Cool, 1989). Estes recursos são "idiossincráticos, não transferíveis e não imitáveis, pois não são negociáveis entre empresas" (Bandeira- 
de-Mello \& Cunha, 2000). Essas características individuais dificultam a imitação dos recursos pelos concorrentes. A heterogeneidade pode ser conservada por meio dos "mecanismos de isolamento", que impedem a imitação de recursos estratégicos pela concorrência, compondo e delineando as decisões estratégicas posteriores da empresa. Estes mecanismos possuem relação com as características inerentes às organizações, como a cultura, a heterogeneidade, a ambiguidade causal, a história da firma, a complexidade social e a singularidade de recursos (Dierickx; Cool, 1989).

Em seu trabalho intitulado "Resource-Based Theory: creating and sustaining competitive", publicado em 2007, Barney fez uma revisão nas características definidas no modelo VRIS para obtenção da vantagem competitiva. Nesta revisão, o elemento Substituibilidade (S) é suprimido, incorporado ao atributo imitabilidade imperfeita, e alterado pela premissa Organização (O), dando origem ao modelo VRIO (Gonçalves, Coelho, \& Souza, 2011). O novo atributo permeia a capacidade da empresa de explorar e converter recursos considerados fontes de vantagem competitiva, em efetiva vantagem competitiva, ou seja, a empresa deve estar devidamente organizada para utilizar estrategicamente seus recursos. Desse modo, o atributo (O)rganização, revelando sua ênfase intangível, reforça o conjunto dos outros atributos - VRI, relacionando-os estrategicamente. O autor cita como elementos mais relevantes para a organização aqueles ligados à estrutura, entre eles sua definição hierárquica, sistemas de controle formais e informais e políticas de remuneração (Barney \& Clark, 2007).

A universidades, em especial as federais, são organizações complexas, com características específicas, que enfrentam, como outras organizações, desafios permanentes, exigindo um alto nível de especialidade funcional em uma gestão que a visualize como parte do processo que envolve sua permanência no mercado (Tachizawa \& Andrade, 2006).

Desse modo, pressupõe-se que a teoria da RBV poderá apoiar a gestão das universidades federais na concepção e elaboração de estratégias que permitirão a elas utilizar seus recursos de forma eficaz, especialmente aqueles alocados para a pósgraduação, foco deste trabalho.

\section{METODOLOGIA}

A pesquisa classifica-se, quanto à abordagem, como qualitativa e exploratória, pois objetiva conhecer a variável de estudo tal como se apresenta, seu significado e o contexto na qual ela se insere, pressupondo que o comportamento humano é melhor compreendido no contexto social onde ocorre (Queiróz, 1992). 
O procedimento de condução escolhido para a pesquisa é classificada como estudo de caso, definido por Yin (2015, p. 17) como uma "inquirição empírica que investiga um fenômeno contemporâneo dentro de um contexto da vida real, quando a fronteira entre o fenômeno e o contexto não é claramente evidente e onde múltiplas fontes de evidência são utilizadas". A utilização de estudos de caso como estratégia para a pesquisa, possibilita ao pesquisador explorar profundamente o objeto em estudo, analisando-o, após a coleta de dados realizada por meio de procedimentos específicos, considerando o tempo e a atividade (Creswell, 2010).

Nessa pesquisa, será utilizado o modelo de casos múltiplos holísticos, pois uma única questão será analisada em três universidades federais. A opção pelo estudo de caso, em especial ao de múltiplos casos, recai na possibilidade que este método de análise proporciona tornar os resultados mais robustos, resguardando suas especificidades, tornando-os passíveis de uma "generalização analítica", definida por Yin (2015) como a aplicação do estudo de caso em contextos diferentes, a partir de proposições teóricas geradas pela pesquisa.

A definição das unidades de análise considerou o baixo número de programas ofertados pelas regiões norte e centro-oeste, bem como o reduzido montante destinado a essas regiões para fomento e desenvolvimento da pós-graduação. Desse modo, definiu-se como objeto de estudo para essa pesquisa, a Universidade Federal de Mato Grosso/UFMT, a Universidade Federal do Amazonas/UFAM e a Universidade Federal do Mato Grosso do Sul/UFMS. A escolha dessas universidades justifica-se pelas características que elas apresentam, vez que, ou pertencem à mesma região geográfica (região centro-oeste) ou a mesma região legal (Amazônia Legal), compartilhando as mesmas dificuldades relacionadas à cultura, clima, infraestrutura, atração de pessoal qualificado, entre outras características específicas dessas regiões.

Para a pesquisa foram utilizados dados secundários e primários. A coleta dos dados secundários foi realizada por meio de pesquisa nos sites das universidades investigadas, bem como em sites de instituições que coletam dados da pós-graduação stricto sensu das universidades públicas, como a CAPES e o INEP. A busca pelos dados secundários envolveu análises de dados disponibilizados em relatórios de gestão e Planos de Desenvolvimento Institucional/PDI elaborados pelas investigadas, assim como o uso de ferramentas como a Plataforma Sucupira e GeoCAPES.

Para a coleta dos dados primários, foram realizadas seis entrevistas com gestores institucionais da pós-graduação stricto sensu das universidades pesquisadas, com mandatos vigentes no período de 2008 a 2017, guiada por um roteiro semiestruturado, buscando apreender as estratégias e a forma de alocação de recursos institucionais 
utilizada por cada gestão na busca pelo desenvolvimento da pós-graduação stricto sensu da instituição nas avaliações CAPES.

A análise dos dados coletados, amparada na teoria RBV, foi realizada procurando identificar como as pró-reitorias de pós-graduação alocaram recursos institucionais em busca do desenvolvimento da pós-graduação stricto sensu.

\subsection{CARACTERIZAÇÃO DAS UNIDADES DE ANÁLISE}

A escolha de universidades públicas federais sediadas nas regiões norte e centrooeste como unidades de análise da pesquisa, atende ao preceito de observar regiões que buscam dirimir a assimetria regional e intrarregional na oferta de programas de pós-graduação stricto sensu, por meio da adoção de estratégias e alocação de recursos institucionais voltados para o fortalecimento da pós-graduação stricto sensu.

\subsubsection{Universidade Federal de Mato Grosso}

A Fundação Universidade Federal de Mato Grosso foi criada por meio da lei no 5647, sancionada em 10 de dezembro de 1970, portanto, durante o governo militar. Sua constituição foi composta, inicialmente, pela Faculdade de Direito de Mato Grosso, fundada em 1957, e pelo Instituto de Ciências e Letras de Cuiabá, cujas atividades iniciaram em 1966 (Veloso, 2000).

Em 2017, a UFMT ofertou 6.168 vagas para ingresso em 106 cursos de graduação, na modalidade presencial, e 947 vagas em 43 programas de pósgraduação stricto sensu, distribuídas nos cinco campus da instituição. Sua população universitária contava com 27.022 indivíduos, aproximadamente, dispostos entre discentes da graduação presencial (19.983), da graduação a distância (1.408), discentes da pós-graduação stricto sensu (2.175), residência médica (149), docentes (1.823) e técnicos administrativos (1.574) (PROPLAN, 2018a).

Na última avaliação quadrienal, cujos resultados foram divulgados em 2017, pode-se avaliar que a UFMT avança na pós-graduação stricto sensu, ofertando 16 cursos de doutorado e 47 cursos de mestrado, distribuídos nos campus de Cuiabá, Araguaia, Rondonópolis e Sinop.

Além do crescimento quantitativo, a UFMT está alcançando um crescimento qualitativo na pós-graduação stricto sensu, conseguindo avançar na consolidação de seus programas. Corroborando essa análise, a instituição alcançou $12 \%$ de seus programas com nota 5 e 35\% dos programas com nota 4 na avaliação quadrienal, conceitos atribuídos a cursos "consolidados" ou "em consolidação (Maccari, Lima, \& Riccio, 2009; Brasil, 2018). 


\subsubsection{Universidade Federal de Mato Grosso do Sul}

A Fundação Universidade Federal de Mato Grosso do Sul/UFMS teve sua origem em 1962, com a criação da Faculdade de Farmácia e Odontologia de Campo Grande. No entanto, sua criação foi efetivada com a publicação da lei complementar no 31 , de 11 de outubro de 1977, que estabeleceu a divisão do estado de Mato Grosso, criando o estado de Mato Grosso do Sul. Em seu artigo 39, a lei previa a federalização da Universidade Estadual de Mato Grosso, efetivada em 1979, com a publicação da lei Federal $n^{\circ}$ 6.674, passando a instituição a denominar-se Fundação Universidade Federal de Mato Grosso do Sul/UFMS, sediada na, agora, capital do estado de Mato Grosso do Sul, Campo Grande.

Em 2017, as unidades acadêmicas da UFMS ofertaram 5.190 vagas em 112 cursos de graduação e 1.116 vagas em 45 programas de pós-graduação stricto sensu, distribuídas em 42 cursos de mestrado e 15 cursos de doutorado. Sua população universitária é de aproximadamente 24.876 pessoas, integrando 18.890 alunos de graduação, 2.244 discentes de pós-graduação stricto sensu, 1803 docentes e 1939 técnicos administrativos (CPA, 2018; PROPLAN, 2018).

O resultado da avaliação quadrienal (2013-2016), divulgado em 2017, demonstrou que a UFMS está buscando consolidar, além de expandir sua pósgraduação stricto sensu. Dos 45 programas avaliados, um alcançou o conceito 6; cinco programas atingiram o conceito $5 ; 17$ obtiveram o conceito $4 ; 21$ foram avaliados com o conceito 3, e um programa não alcançou a nota mínima para continuar funcionando, sendo avaliado com o conceito 2 e tendo suas atividades desativadas. No geral, o resultado foi positivo, pois $52 \%$ dos programas da instituição foram avaliados com conceitos que os definem como "em consolidação", "consolidados" ou de "excelência" (Maccari, Lima, \& Riccio, 2009; Brasil, 2018).

\subsubsection{Universidade Federal do Amazonas}

A história da Universidade Federal do Amazonas inicia em 1909, com a implantação da Escola Universitária Livre de Manaus, primeira a ofertar cursos de nível superior na região, destinados à instrução militar de oficiais da Guarda Nacional e de outras milícias, abertos a qualquer cidadão. Somente em 20 de junho de 2002, com a publicação da Lei no. 10.468, é que a universidade recebeu a denominação de Universidade Federal do Amazonas/UFAM (UFAM, 2019).

A UFAM ofertou, em 2017, 5.506 vagas para 116 cursos de graduação e mais de 854 vagas em 40 programas de pós-graduação stricto sensu, sendo que 37 desses eram cursos de mestrado acadêmico, 10 de mestrado profissional e 16 de doutorado. 
Nesse mesmo ano, a população universitária da instituição (constituída por 27.757 alunos matriculados em cursos de graduação presencial, 1.629 alunos matriculados na graduação a distância, 2.126 discentes de pós-graduação, 1.829 docentes e 1.721 técnicos administrativos) atingiu cerca de 35.062 pessoas, contando com o apoio de laboratórios, biotério, fazenda experimental, museu e um centro de artes para o desenvolvimento das atividades acadêmicas (PROPLAN, 2017).

Os programas de pós-graduação stricto sensu da UFAM não obtiveram o desempenho esperado na avaliação quadrienal que trouxe como resultado três programas com a atribuição do conceito 2, encerrando suas atividades; 21 avaliados com conceito 3; 18 que alcançaram o conceito 4 e apenas dois com a atribuição do conceito 5. No entanto, após analisar os resultados, a UFAM fortaleceu sua pósgraduação e finalizou o ano de 2017 com 42 programas de pós-graduação, sendo 15 doutorados, 29 mestrados acadêmicos e 9 mestrados profissionais (PROPLAN, 2017).

\section{APRESENTAÇÃO E ANÁLISE dOS RESULTADOS}

O apoio institucional dedicado à pós-graduação stricto sensu na UFMT contou com a alocação de recursos humanos, físicos, tecnológicos, organizacionais e financeiros necessários para implementar as ações que envolveram a busca pela consolidação dos cursos de pós-graduação stricto sensu existentes na instituição; o estímulo a proposição de novos cursos de mestrado e/ou doutorado, a qualificação do corpo docente, com a ampliação de professores doutores aptos a atuarem na pósgraduação stricto sensu; a realização de avaliação institucional dos programas de pósgraduação stricto sensu em andamento; o acompanhamento individualizado das demandas dos coordenadores de pós-graduação stricto sensu e a divulgação dos programas existentes na instituição (CPA, 2010).

Os recursos financeiros foram disponibilizados para a pós-graduação da UFMT por meio de dotação orçamentária específica direcionada à Pró-reitoria de Ensino de Pós-graduação/PROPG. Esses recursos foram investidos, prioritariamente, no custeio de passagens e hospedagens para membros externos de bancas de defesa de mestrado ou doutorado. O investimento nesse custeio favoreceu o planejamento dos programas de pós-graduação stricto sensu para o uso do recurso financeiro de custeio recebido anualmente da CAPES, o Programa de Apoio à Pós-graduação/PROAP, em demandas específicas do programa na busca de um melhor desempenho na avaliação da CAPES. O relato da pró-reitora reforça a importância dessa ação para o desenvolvimento dos programas:

"A maior parte desse orçamento era utilizada para o custeio de todas as bancas de defesas. Ninguém foi barrado por querer trazer um professor 
que fosse lá do extremo sul ou do extremo norte do país, todos os professores que desejavam trazer um especialista para suas bancas puderam fazer isso, o que foi muito importante e é uma prática que eu não vejo acontecer em outros locais, pois, normalmente, esses recursos são retirados dos PROAP dos programas. No nosso caso, os PROAPs chegavam limpos para o programa, as despesas com bancas ficavam todas por conta da instituição, e é uma conta enorme. Esse é um ponto fundamental e importantíssimo para que as políticas dos PPGs pudessem funcionar". (UFMT, outubro 2018)

Os recursos financeiros também foram aplicados no custeio de tradução de artigos, aquisição de passagens e concessão de diárias para professores e discentes apresentarem trabalhos em congressos nacionais e internacionais, além de financiar as despesas operacionais dos programas em rede, profissionais, que não recebem auxílio das agências de fomento, e das demandas não subsidiadas no financiamento dos programas interinstitucionais (SECOMM, 2016).

A PROPG também buscou suprir as necessidades dos programas, e da própria pró-reitoria, referente a alocação de recursos humanos. Na pró-reitoria, o quadro de servidores manteve-se estável em relação ao ano de 2008, quando oito servidores atuavam na PROPG, mesmo levando-se em conta as aposentadorias, exonerações, redistribuições e remoções ocorridas até 2017, agora com nove servidores lotados na pró-reitoria (SECOMM, 2016).

Recursos tecnológicos e organizacionais também foram alocados na execução das estratégias adotadas para consolidar a pós-graduação stricto sensu da UFMT. No período em estudo, a PROPG adquiriu, com recursos institucionais, novos equipamentos de informática e mobiliário, que foram distribuídos entre os programas e a pró-reitoria, melhorando as condições de trabalho para os servidores das secretarias e coordenadores dos programas de pós-graduação stricto sensu. Além desses recursos, a instituição investiu no desenvolvimento de sistemas de apoio às atividades administrativas da pós-graduação, na aquisição de servidor de e-mail específico para a pós-graduação, na otimização de rotinas em processos que envolvem a pós-graduação e, especialmente, na mudança na cultura organizacional, fazendo a pós-graduação ser reconhecida como um ambiente de "ensino", passando as atividades desenvolvidas nesse nível de ensino, por força de resolução, a ser contadas no plano de atividades do docente (SECOMM, 2016).

A gestão superior, juntamente com a PROPG e a Secretaria de Relações Internacionais, trabalharam em conjunto com as coordenações dos programas de pósgraduação stricto sensu para implementar parcerias e convênios, nacionais e internacionais. A atenção à internacionalização, no âmbito da UFMT, remonta aos anos de 1980, quando foi instituída uma Assessoria de Relações Internacionais/ARI, tendo 
por meta subsidiar a reitoria na elaboração e execução de políticas que impulsionassem a inserção da instituição no contexto mundial. Em 2008, a ARI propôs à administração superior a reformulação Planejamento Estratégico Participativo, incluindo metas que pudessem fortalecer o processo de internacionalização da instituição nos dez anos seguintes (SECOMM, 2016).

Em 2012, a ARI foi transformada na Secretaria de Relações Internacionais/SECRI, revelando o empenho da gestão superior em atingir as metas propostas para a internacionalização e a pós-graduação stricto sensu. Com o trabalho da SECRI, foram firmadas parcerias com instituições de ensino em mais de 30 países, além de parcerias, nacionais e internacionais, em rede e bilaterais, inserindo a UFMT no cenário internacional como parceira em países de relevância científica como França, Alemanha, Estados Unidos, China, Japão, entre outros. A gestora da PROPG ressalta a importância dessas parcerias para o desenvolvimento da pós-graduação stricto sensu da instituição:

\begin{abstract}
"Esses intercâmbios são muito importantes porque nucleamos; no momento em que vem pessoas de outro país ou outros estados brasileiros para nossos programas, elas irão levar para suas instituições de origem o conhecimento adquirido aqui no grupo de pesquisa ao qual ela pertenceu e do qual poderá continuar a fazer parte. Isso facilita a relação entre professores das instituições envolvidas no processo". (UFMT, outubro 2018)
\end{abstract}

A Pró-reitoria de Pesquisa e Pós-graduação/PROPP, da UFMS, recebeu a alocação de recursos financeiros institucionais específicos, para serem utilizados na busca pelo cumprimento das metas estabelecidas no PDI (PROPLAN, 2015).

Os recursos financeiros disponibilizados foram alocados no custeio de despesas para publicação de artigos em revistas qualificadas nos estratos mais elevados do sistema QUALIS, passagens e diárias para docentes e discentes apresentarem trabalhos em congressos, além de aquisição e manutenção de equipamentos e softwares demandados pelos programas de pós-graduação stricto sensu, viabilizando a modernização e instrumentalização dos laboratórios utilizados nas pesquisas desenvolvidas na instituição (PROPLAN, 2015).

O gestor da PROPP, em seu relato, explicita a necessidade de ampliar a participação dos professores doutores da instituição na pós-graduação stricto sensu:

"Utilizamos estes recursos para publicação de artigos em revistas qualificadas, pelo menos QUALIS B1, para que professores que não fazem parte dos programas de pós-graduação pudessem ir apresentar trabalhos, de forma a incentivar essas pessoas a alcançarem a publicação necessária para participar de um programa de pósgraduação. Isso porque temos aproximadamente um terço dos nossos doutores que não participam dos nossos programas e esse número é 
muito alto, precisamos fomentar a participação desses docentes nos nossos programas". (UFMS, outubro 2018)

Quanto aos discentes, a PROPP destinou pela primeira vez no período em estudo, recursos financeiros institucionais para concessão de bolsas de mestrado e doutorado, além de incentivar a produção científica dos mesmos com o custeio de diárias e passagens para apresentação de trabalhos em congressos (PROPLAN, 2015b). Estes incentivos, colaboraram para que a universidade atingisse a meta proposta em seu PDI de aumentar a taxa de sucesso na pós-graduação, bem como a qualidade de sua produção científica.

No que tange aos recursos físicos e tecnológicos, a gestão da UFMS investiu na modernização e manutenção de sua infraestrutura. O gestor da PROPP afirma que, por meio de seu orçamento institucional, envidou esforços para adquirir equipamentos para secretarias e laboratórios, demandados pelos coordenadores dos programas de pós-graduação stricto sensu. Todos os campus foram contemplados com a instalação de, pelo menos, uma sala de vídeo conferência. Também foram viabilizados pontos de acesso à rede de internet sem fio, conhecidos como hot-spot, nas unidades acadêmicas da sede e dos campus do interior (PROPLAN, 2014).

A internacionalização na UFMS é reconhecida como estratégica na busca pela consolidação dos programas de pós-graduação stricto sensu, pois proporcionam oportunidades de aperfeiçoamento profissional e qualificação aos estudantes de graduação, pós-graduação e egressos. Esse pensamento se reflete na declaração da missão da instituição, explicitada no PDI do período 2010-2014:

"Desenvolver, difundir e socializar o conhecimento por meio do ensino, da pesquisa, da extensão e da prestação de serviços e promover a formação integral e permanente dos cidadãos, preparando-os para que possam intervir e atuar com dinamismo no processo de desenvolvimento local, regional, nacional e internacional".

No entanto, no âmbito da pós-graduação stricto sensu, a grande maioria dos intercâmbios internacionais são acertados por meio de relações pessoais entre professores das duas instituições, sem acordo formal ou firmação de convênio.

"A universidade é bem internacionalizada, mas pontualmente, isso foi diagnosticado na construção do projeto para o CAPESPrInt. Precisamos institucionalizar essas ações isoladas de grupos de pesquisa ou pesquisadores, incluindo-os no projeto institucional de internacionalização [...]". (UFMS, outubro 2018)

Nesse sentido, a PROPP incentivou a participação de professores e alunos em intercâmbios e programas de mobilidade em parcerias firmadas com instituições nacionais e estrangeiras, além da participação no programa governamental "Ciências 
sem Fronteiras", fortalecendo a nucleação com a troca de experiências vivenciadas, ampliando a inserção internacional da UFMS em países como Argentina, Espanha, Estados Unidos, França, Itália, Portugal, República Tcheca, entre outros (PROPLAN, 2011).

Os resultados obtidos com a política de internacionalização da UFMS são considerados positivos pelo gestor da PROPP, que destaca a aprovação do projeto de internacionalização proposto no Programa Institucional de Internacionalização/CAPESPrInt:

"Posso avaliar que os resultados obtidos com a política de internacionalização implementada pela universidade são bons, tanto é que o projeto CAPES-PrInt foi provado. Nesse projeto mostramos como se dá o resultado da nossa internacionalização e a CAPES, de alguma forma, verificou que nossa internacionalização está funcionando, não da forma ideal, como queremos e como a CAPES quer, mas que se tem algo iniciado e que está dando resultado." (UFMS, outubro, 2018)

O apoio institucional à pós-graduação stricto sensu na UFAM oscilou entre as gestões eleitas para administrar a Pró-reitoria de Pesquisa e Pós-graduação/PROPESP nos períodos de 2009-2013, 2013-2017 e 2017-2021.

No primeiro período de mandato, de julho/2009 a julho/2013, o apoio institucional à pós-graduação pode ser verificado, notadamente, por meio da elaboração, aprovação e implementação de um Programa de Apoio à Consolidação e ao Avanço da Qualidade da Pós-Graduação da Universidade Federal do Amazonas, o PACPG/UFAM.

A gestora da PROPESP, com o apoio da administração superior da universidade, alocou durante sua gestão (2009-2013), recursos financeiros e humanos, conforme sua disponibilidade, para cumprir com as propostas previstas no PACPG/UFAM, observando os princípios norteadores explicitados no PDI 2006-2015.

A alocação dos recursos financeiros foi, prioritariamente, destinada a custear despesas vinculadas à produção científica de docentes e discentes de programas de pós-graduação stricto sensu, disponibilizados por meio de editais de auxílio, aos quais os interessados podiam se candidatar naquele que melhor the conviesse.

A destinação de recursos humanos para atuar na pós-graduação stricto sensu não conseguiu atender a demanda por técnicos administrativos para atuarem na PROPESP ou nas secretarias dos programas de pós-graduação stricto sensu. Quanto à participação dos docentes da instituição nos programas de pós-graduação stricto sensu, a pró-reitoria incentivou a produção científica dos docentes, custeando as despesas para publicação e apresentação de trabalhos em congressos, além de implantar o programa de reserva de vagas nos processos seletivos da pós-graduação, 
ampliando, desse modo, as possibilidades de credenciamento de recém-doutores à pós-graduação stricto sensu, além de impulsionar a produção científica dos programas. Ainda apoiando os programas de pós-graduação stricto sensu, a gestão priorizou a contratação de professores visitantes para atuar, preferencialmente, nesse nível de ensino, ministrando disciplinas, orientando trabalhos de dissertação e tese, além de promover a integração da pós-graduação com a graduação por meio de pesquisas conjuntas (CPA, 2013).

Em relação à alocação dos recursos físicos para a pós-graduação stricto sensu, a gestão da PROPESP atuou junto às unidades acadêmicas para prover os recursos físicos necessários para o pleno desenvolvimento das atividades administrativas, de ensino e pesquisa desenvolvidas no âmbito da pós-graduação stricto sensu (PROPLAN, 2018).

Acerca dos recursos tecnológicos, a UFAM investiu na implantação de uma rede de internet wifi-zone-UFAM, proporcionando o livre acesso a toda comunidade acadêmica, interna e externa, com cobertura de sinal, especialmente, para os blocos de sala de aula. Também foram investidos recursos para a instalação de uma rede de rádios e fibra ótica, especialmente para os blocos de sala de aula que não possuíam infraestrutura de rede. Entre as principais dificuldades para suprir as demandas por recursos físicos e tecnológicos, a gestão da UFAM apresenta o contingenciamento orçamentário, a crescente demanda de serviços devido à expansão verificada nos cursos de graduação e pós-graduação da universidade e a crescente ampliação de gastos com o custeio (como manutenção, vigilância, conservação, entre outros) da universidade (PROPLAN, 2018).

A gestão que atuou na PROPESP entre julho/2013 e julho/2017, entendeu que os resultados obtidos pelos programas de pós-graduação stricto sensu da UFAM na avaliação trienal 2010/2012, divulgada em 2013, não foram satisfatórios e decidiram reformular o PACPG/UFAM (PROPLAN, 2018).

Desse modo, nessa gestão, para a alocação dos recursos financeiros destinados à pós-graduação stricto sensu, a PROPESP solicitou às coordenações dos programas de pós-graduação stricto sensu um planejamento das demandas para esse recurso. A concessão dos recursos priorizou atividades que melhorassem a formação dos discentes, notadamente a produção científica dos programas de pós-graduação stricto sensu (programas Caxiri, Nhengatu e Vamos Publicar!) e bancas de defesa e de qualificação (PROPLAN, 2018).

"Além do orçamento que vem da CAPES, de agências de fomento ou captações, na nossa instituição tínhamos um orçamento específico para a pós-graduação. Antevendo a crise, os cortes iniciais foram muitos na 
PROPESP, fomos recuperando aos pouquinhos o orçamento e criando orçamento para o Vamos Publicar, além de garantir a participação de professores externos em bancas de defesas quando o programa não contava mais com recursos do PROAP." (UFAM1, setembro 2018)

A nova gestão da UFAM efetivou uma reestruturação administrativa, criando, no âmbito da PROPESP, uma Diretoria de Avaliação e Acompanhamento/DAV. Para o devido funcionamento da DAV, servidores foram alocados, incluindo docentes e técnicos administrativos. No entanto, a expansão no número de servidores da próreitoria deveu-se, apenas, no preenchimento dos cargos com função gratificada criados com sua reestruturação, não suprindo, desse modo, as necessidades da próreitoria. A PROPESP considerou estratégico solucionar a demanda por técnicos administrativos para as secretarias dos programas de pós-graduação stricto sensu, bem como o incentivo à participação de jovens doutores nos programas de pósgraduação stricto sensu da instituição.

"Foi utilizado como estratégia visitas aos programas, onde era realizado um levantamento de demandas e tentativas de solução dessas demandas, que iam desde o apoio técnico para secretarias, compra de equipamentos, manutenção, como também ao incentivo para publicações de livros, artigos, entre outros." (UFAM1, setembro 2018)

A alocação de recursos físicos para a pós-graduação stricto sensu a responsabilidade persistiu no âmbito das unidades acadêmicas, existindo, no entanto, alguns prédios para uso exclusivos de programas de pós-graduação stricto sensu. Em relação aos recursos tecnológicos, a gestão superior da UFAM investiu no projeto UFAM Digital, que visa integrar os diversos sistemas de gestão da informação e comunicação utilizados na instituição, bem como reduzir o uso de papel em todos processos e trâmites administrativos operacionalizados na UFAM (PROPLAN, 2018).

Outra melhoria relacionada a alocação tanto de recursos físicos quanto tecnológicos, foi citada pelo gestor da PROPESP, salientando a instalação de "salas de vídeo conferência, para que as qualificações pudessem ser feitas a distância e as vindas de professores ocorressem somente na defesa", tanto na sede quanto nos campus do interior, para uso dos programas de pós-graduação stricto sensu (UFAM1, setembro 2018).

Diante dos contingenciamentos orçamentários implementados pelo governo federal, iniciados em 2013, a administração superior da UFAM implantou o Programa Intensivo de Otimização Financeira, priorizando atividades consideradas essenciais para o funcionamento da instituição, como o pagamento de bolsas estudantis, refeições subsidiadas, serviços de limpeza, conservação e vigilância. Outras dificuldades como greves de servidores e redução do quadro funcional, devido a 
aposentadorias e exonerações, influenciaram no desempenho das atividades da UFAM, bem como no total cumprimento das metas previstas no PDI para o período (PROPLAN, 2018).

Em julho de 2017 uma nova gestão assumiu a administração superior da UFAM e retomou o plano de atividades previstas no Programa de Apoio à Consolidação e ao Avanço da Qualidade da Pós-graduação/PACPG/UFAM, implementado em 2012 e, posteriormente, interrompido por quatro anos. Nesse sentido, a alocação de recursos financeiros, humanos, físicos e tecnológicos institucionais na nova gestão atenderá a pós-graduação stricto sensu, conforme planejamento realizado para cumprimento das metas previstas no PDI 2016-2025, alinhado com as fases de execução do PACPG/UFAM, incentivando a produção científica, a modernização e ampliação das instalações utilizadas nas atividades dos programas de pós-graduação stricto sensu (tais como a manutenção da infraestrutura física por meio de pequenas obras que envolvam reforma; adaptação, aquisição ou reposição de materiais) e continuidade na política de qualificação dos servidores da instituição, buscando ampliar e aprimorar a participação desses servidores, especialmente os docentes, nos programas e nas atividades que envolvem a pós-graduação stricto sensu (PROPLAN, 2017).

Na UFAM, no período de 2009 a 2017, as ações de internacionalização experiências no âmbito da pós-graduação, em sua essência, são desenvolvidas diretamente pelos docentes credenciados nos programas de pós-graduação stricto sensu e, quando institucionalizadas, são intermediadas pela Assessoria de Relações Institucionais e Internacionais/ARII.

"[...] identificamos a existência de muitas parcerias, no entanto, são parcerias individualizadas, no contexto de pesquisador ou do programa. Estamos trabalhando nessa questão de institucionalizar nossos projetos internacionais e criando um ambiente de internacionalização na pósgraduação, tradução das páginas, tradução de todos os ambientes da universidade para linha inglesa, enfim, estabelecendo várias metas para poder internacionalizar, incluindo disciplinas em língua estrangeira para estreitar laços com os outros países. " (UFAM, outubro 2018)

Nesse período, para incentivar a ampliação das ações de internacionalização, a PROPESP buscou parcerias com instituições de ensino estrangeiras renomadas, tendo como meta que cada programa de pós-graduação stricto sensu da UFAM firmasse parceria com pelo menos três dessas universidades, o que contribuiria para a elevação da qualidade no ensino e na pesquisa desenvolvidas no âmbito dos programas, além de fortalecer e divulgar, no cenário internacional, a imagem dos programas envolvidos (PROPLAN, 2018). 
O conjunto de recursos institucionais alocados para consolidar e/ou expandir a pós-graduação stricto sensu nas universidades federais foi considerado relevante pelos gestores entrevistados, os quais destacaram, unanimemente, os recursos humanos como essenciais nesse processo de desenvolvimento. Dentre os recursos humanos, os gestores destacaram a importância de se poder contar com o apoio de técnicos qualificados para auxiliar nas rotinas administrativas. Os docentes e discentes também foram citados, especialmente acerca do nível de seu comprometimento com as atividades da pós-graduação. A teoria RBV, defende que os recursos nem sempre são passíveis de transferência ou imitação pela concorrência (Barney, 1991). Isso se confirma, por exemplo, com os recursos humanos qualificados das universidades analisadas, cujo capital intelectual é único e de difícil imitação, revelando-se uma vantagem competitiva para suas instituições quando bem explorados por ela, especialmente para os programas de pós-graduação stricto sensu.

Os recursos financeiros, especificamente os institucionais, foram citados como o segundo recurso mais importante do processo de desenvolvimento da pós-graduação stricto sensu. Esses recursos devem ser aplicados em programas de incentivo à produção científica (pagamento de inscrição, diárias e passagens para participação em eventos, tradução, revisão de artigos, entre outras), auxílio à promoção de eventos científicos, programas institucionais de bolsas de mestrado-doutorado e pósdoutorado, além de subsidiar atividades que busquem melhorar a formação do pósgraduando (coleta de dados, aulas de campo, entre outras), pois seu retorno influencia diretamente nos quesitos utilizados para analisar o desenvolvimento dos programas no período avaliado pela CAPES.

Ferraz, Maccari, Quoniam, Silva, \& Modkovski (2017) afirma que o acompanhamento constante da produção científica dos docentes envolvidos com a pós-graduação, pois esta representa uma estratégia não só para a busca de melhor conceito do programa junto à Capes, mas também para manutenção de um conceito já conquistado, expressando a excelência do programa de pós-graduação em sua área de atuação. Assim, a alocação dos recursos institucionais, especialmente aqueles que foram identificados como fonte de vantagem competitiva pelos respondentes, devem amparar as atividades desenvolvidas pela pró-reitoria e pelos programas buscando melhores resultados na avaliação CAPES.

\section{CONSIDERAÇÕES FINAIS}

O crescimento constante da pós-graduação stricto sensu brasileira e seu reconhecimento como principal agente de formação de profissionais altamente qualificados, destacam sua contribuição para desenvolvimento tecnológico, científico 
e cultural do país (CAPES, 2010; Maccari, 2008b). Em 2017, as universidades federais foram responsáveis pela oferta de aproximadamente $57 \%$ dos programas de pósgraduação stricto sensu do país, corroborando a preocupação do VI PNPG (20112020), que ressaltou em seu texto a importância de se investir na infraestrutura utilizada pelos programas de pós-graduação stricto sensu dessas instituições para desenvolver suas pesquisas.

Nesse trabalho, procurou-se analisar como as pró-reitorias de pós-graduação de universidades federais alocaram recursos institucionais em busca do desenvolvimento de seus programas de pós-graduação stricto sensu.

Com os resultados da pesquisa, foi possível concluir ser imprescindível para a expansão e/ou consolidação da pós-graduação stricto sensu, ofertadas pelas universidades federais, o seu reconhecimento pela administração superior como estratégica para o desenvolvimento institucional, fazendo-a constar no PDI e, respectivamente, nos planejamentos estratégicos de curto/médio prazos propostos pela gestão. Esse registro asseguraria a alocação de seus recursos institucionais utilizando sua experiência acumulada e de acordo com as políticas institucionais definidas em seu planejamento estratégico.

Esse trabalho limitou-se a investigar o uso dos recursos institucionais para a execução de estratégias voltadas para o desenvolvimento da pós-graduação stricto sensu em universidades federais brasileiras. Assim, reconhecendo que esse estudo proporciona somente uma generalização analítica, por ser fruto de um estudo de caso múltiplo (Yin, 2015), é possível que os achados dessa pesquisa, respeitando as especificidades de cada instituição, contribua com outras universidades, especialmente as federais, no processo de expansão e consolidação de sua pós-graduação stricto sensu na avaliação periódica conduzida pela CAPES.

Desse modo, esse trabalho apenas iniciou uma investigação em um amplo campo que necessita ser explorado em seus diversos aspectos, de forma a ampliar o reconhecimento institucional das atividades desenvolvidas no âmbito da pósgraduação stricto sensu. Assim, propõe-se a continuidade da pesquisa, agora incluindo na análise os recursos externos descentralizados para as universidades para auxiliar no custeio das atividades de ensino e pesquisa desenvolvidas nos programas de pósgraduação stricto sensu, ou ainda, recebidos por meio de aprovação para financiamento de projetos de pesquisadores vinculados a esse nível de ensino.

\section{REFERÊNCIAS}

ACS/CAPES; Assessoria de Comunicação Social da Capes. (2011). Revista Capes 60 anos. Revista Comemorativa, 56. 
Almeida Júnior, A., Sucupira, N., Salgado, C., Barreto Filho, J., Silva, M. R. e, Trigueiro, D., Maciel, R. (2005). Documento Parecer CFE n 977/65. Revista Brasileira de Educação, (30), 162-173. Retrieved from http://www.redalyc.org/articulo.oa?id $=27503014$

Balbachevsky, E. (2005). A pós-graduação no Brasil: novos desafios para uma política bem sucedida. Os Desafios Da Educação No Brasil. Rio de Janeiro: Nova Fronteira, 1, 285-314.

Bandeira-de-Mello, R., \& Cunha, C. J. C. de A. (2000). A Natureza e a Dinâmica das Capacidades Organizacionais no Contexto Brasileiro: Uma Agenda para Pesquisas Sobre a Vantagem Competitiva das Empresas Brasileiras. Department of Management and Economics, 440.

Bardin, L. (2016). Análise de Conteúdo. São Paulo: Edições 70.

Barney, J. B. (1986). Organizational Culture: Can It Be a Source of Sustained Competitive Advantage? Academy of Management Review1, 11(3), 656-665.

Barney, J. B. (1991). Firm Resources and Sustained Competitive Advantage. Journal of Management, 17(1), 99-120.

Barney, J. B., \& Clark, D. N. (2007). Resource-Based Theory: creating and sustaining competitive. New York: Oxford University.

Belloni, J. A. (2000). Uma metodologia de avaliação da eficiência produtiva de universidades federais brasileiras. Universidade Federal de Santa Catarina.

Boratim, R. J. (2014). Coordenação de aperfeiçoamento de pessoal de nível superior: agente regulador do campo da pós-graduação stricto sensu no Brasil. Semina: Ciências Sociais e Humanas, 35(2), 49. https://doi.org/10.5433/16790383.2014v35n2p49

Brasil. (2014). Planejando a Próxima Década Conhecendo as 20 Metas do Plano Nacional de Educação. Secretaria de Articulação Com Os Sistemas de Ensino (MEC/ SASE), $62 . \quad$ Retrieved from http://pne.mec.gov.br/images/pdf/pne_conhecendo_20_metas.pdf

Brasil. (2018a). Decreto $\mathrm{n}^{\circ}$ 74.299, de 18 de Julho de 1974. Retrieved February 22, 2018, from http://www2.camara.leg.br/legin/fed/decret/1970-1979/decreto74299-18-julho-1974-422808-publicacaooriginal-1-pe.html

Brasil. (2018b). LEI No 4.024 - LEI DE DIRETRIZES E BASES DA EDUCAÇÃO. Retrieved $\quad$ February 1, 2018, from https://www.planalto.gov.br/ccivil_03/Leis/L4024.htm\#art120

Retrieved

BRASIL, A. S. (2017). Ângela Portela critica cortes na educação superior. https://www12.senado.leg.br/noticias/materias/2016/08/24/angela-portela-criticacortes-na-educacao-superior

CAPES, C. de A. de P. de N. S. (n.d.). História e missão. Retrieved February 5, 2017, from http://www.capes.gov.br/historia-e-missao 
CAPES, C. de A. de P. de N. S. (2010). Plano Nacional de Pós-Graduação - PNPG 2011-2020 (Vol. 1). Brasília: CAPES. Retrieved from http://www.capes.gov.br/images/stories/download/Livros-PNPG-Volume-I-Mont.pdf

CAPES, C. de A. de P. de N. S. (2017). RELATÓRIO FINAL 2016 - Sumário Executivo, 1-43.

CPA, C. P. de A. (2010). RELATÓRIO DE AVALIAÇÃO INSTITUCIONAL ANO DE EXERCÍCIO : 2010. Cuiabá/MT.

CPA, C. P. de A. (2013). RELATÓRIO DE AUTOAVALIAÇÃO 2012. Manaus/AM.

CPA, C. P. de A. (2018). Relatório de Autoavaliação Insitucional - Triênio 20152017 UFMS. Campo Grande/MS.

Creswell, J. W. (2010). Projeto de Pesquisa: métodos qualitativo, quantitativo e misto. Porto Alegre: Armed.

Cury, C. R. J. (2005). Quadragésimo ano do parecer CFE n o 977 / 65. Revista Brasileira de Educação, 1931(30), 7-20. https://doi.org/10.1590/S141324782005000300002

Dierickx, I.; Cool, K. (1989). Asset stock accumulation and sustainability of competitive advantage. Management Science, 33(12).

Ferraz, R. R. N., Maccari, E. A., Quoniam, L., Silva, M. V. C. da, \& Modkovski, A. F. (2017). Planejamento anual e quadrienal de prestação de contas à Capes por meio da ferramenta computacional Scriptsucupira. Revista Brasileira de Pós-Graduação, 14(0), 1-25. https://doi.org/10.21713/2358-2332.2017.V14.1320

França, I. A. (2012). a Gestão Da Pós-Graduação No Brasil Através De Seus Planos Nacionais: Os Desafios Dos Gestores De Cursos. Revista Gestão Universitária Na América Latina, 5, 43-67.

Gonçalves, C. A., Coelho, M. de F., \& Souza, É. M. de. (2011). VRIO : Vantagem competitiva sustentável pela organização. Revista Ciências Da Administração, 17(3), 819-855.

Maccari, E. A., Lima, M. C., \& Riccio, E. L. (2009). Uso do sistema de avaliação da CAPES por programas de pós-graduação em administração no Brasil. Revista de Ciências da Administração, 11(25), 68-82. Retrieved from https://periodicos.ufsc. br/index.php/adm/article/view/2175-8077.2009v11n25p68

Maccari, E. A., Rodrigues, L. C., Alessio, E. M., \& Quoniam, L. M. (2008). Sistema de avaliação da pós-graduação da Capes: pesquisa-ação em um programa de pósgraduação em Administração. Revista Brasileira de Pós-Graduação, 5(9), 171-205. https://doi.org/10.21713/2358-2332.2008

Mendonça, A. (2000). A Universidade no Brasil. Revista Brasileira de Educação, 14(2), 131-150.

Oliveira, S. S. B. de, Oliveira Filho, E. C. de, \& Bentes, A. do N. (2014). A elevação da qualidade da pós-graduação: uma proposta da Universidade Federal do Amazonas. Revista Brasileira de Pós-Graduação, 11(23), 107-126. Retrieved from 
http://ojs.rbpg.capes.gov.br/index.php/rbpg/article/view/524

Pereira, M. S., \& Forte, S. H. A. C. (2008). Visão baseada em recursos nas instituições de ensino superior de Fortaleza: uma análise ex-ante e ex-post à LDB/ 96. Revista de Administração ContemporâNea, 12(1), 107-129. https://doi.org/10.1590/S1415-65552008000100006

Peteraf, M. (1993). The cornerstones of competitive advantage: a resource based-view. Strategic Management Journal, 14, 179-191.

Porter, M. (1980). Competitive strategy. New York: Free Press.

PROPLAN, P. de P. (2018a). Anuário Estatístico 2018 Ano Base 2017. Cuiabá. Retrieved from http://www1.ufmt.br/ufmt/un/secao/14111/anuarioestatistico

PROPLAN, P. de P. (2018b). Relatório de Gestão do exercício de 2017. Cuiabá.

PROPLAN, P. de P. e D. I. (2017). RELATÓRIO DE GESTÃO 2017. Manaus/AM.

PROPLAN, P. de P. e D. I. (2018). RELATÓRIO DE GESTÃO CONSOLIDADO 20092017. Manaus/AM.

PROPLAN, P. de P. e O. (2011). Plano de Desenvolvimento Institucional 2010/2014 Realinhado. Campo Grande/MS.

PROPLAN, P. de P. e O. (2014). Relatório de Avaliação - Plano de Desenvolvimento Institucional 2010/2014. Campo Grande/MS.

PROPLAN, P. de P. e O. (2015a). Plano de desenvolvimento institucional 20152019. Campo Grande/MS.

PROPLAN, P. de P. e O. (2015b). Relatório de Gestão - Exercício 2014. Campo Grande/MS.

PROPLAN, P. de P. O. e F. (2018). Avaliação do PDI 2015-2019. Campo Grande/MS.

Queiróz, M. de P. (1992). O pesquisador, o problema da pesquisa, a escolha de técnicas: algumas reflexões. Lang, ABSG, Org. Reflexões Sobre a Pesquisa Sociológica. São Paulo, Centro de Estudos Rurais e Urbanos, 13-29.

Ribeiro, R. J. (2007). Solidariedade e cooperação na avaliação da pós-graduação. CAPES. Retrieved from http://www.capes.gov.br/avaliacao/permanencia-no-snpgavaliacao/avaliacoes-anteriores/91-conteudo-estatico/avaliacao-capes/6878-artigos

Ribeiro, R., Rossetto, C. R., \& Verdinelli, M. A. (2011). Comportamento estratégico da empresa e a visão baseada em recursos: um estudo no setor varejista de material de construção. Gestão \& Produção, 18(1), 175-192. https://doi.org/10.1590/S0104-530X2011000100013

SECOMM, S. de C. e M. (2016). Relatório de Gestão 2008-2016. Cuiabá.

Siqueira, E. M., Dourado, N. S., \& Ribeiro, R. S. (Eds.). (2011). Universidade Federal de Mato Grosso: 40 anos de História (1970-2010). Cuiabá: EdUFMT. 
Tachizawa, T., \& Andrade, R. O. B. (2006). Gestão de instituições de ensino. (4th ed.). Rio de Janeiro: FGV editora.

UFAM, U. F. do A. (2019). História da Ufam. Retrieved January 15, 2019, from https://ufam.edu.br/historia-da-ugm

Veloso, T. C. M. A. (2000). A evasão nos cursos de graduação da Universidade Federal de Mato Grosso, campus universitário de Cuiabá 1985/2 a 1995/2 - um processo de exclusão. Universidade Federal de Mato Grosso.

Wernerfelt, B. (1984). A Resource based view of the firm. Strategic Management Journal, 5(2), 171-180. https://doi.org/10.1002/smj.4250050207

Yin, R. K. (2015). Estudo de caso: planejamento e métodos. Porto Alegre: Bookman. Retrieved from https://books.google.com/books?hl=en\&lr=\&id=EtOyBQAAQBAJ\&oi=fnd\&pg=PR1\&d $\mathrm{q}=\mathrm{YIN},+\mathrm{R} .+\mathrm{K} .+$ Estudo+de+caso: + planejamento+e+métodos. +3. +ed. +Porto+Alegr e:+Bookman,+2005\&ots=-jcgjoEZAB\&sig=0oGivamB3VY-ngEmK57_P2VnR1Y 